\title{
DIAGNOSIS
}

\section{Programmed probiotics light up liver cancer in urine}

Researchers have harnessed synthetic biology to establish a novel method to detect the presence of liver tumours. By engineering probiotic bacterial strains to deliver a diagnostic readout that can be assessed in urinary samples, a new noninvasive approach to cancer detection has been developed.

Previous work had investigated nanomaterials as a method to detect gastrointestinal disease (such as colorectal cancer). "We had been seeking to increase accumulation of such diagnostic nanoparticles in tissues and design systems that could amplify their recruitment," explains author Sangeeta Bhatia. "One idea we had was that a living vehicle would be able to self-amplify [the nanomaterial] simply by multiplication." Engineered bacteria being one such approach given that bacteria have been shown to selectively grow inside tumours.

Bhatia and colleagues engineered a widely used probiotic, Escherichia coli Nissle 1917, to highly express a lacZ

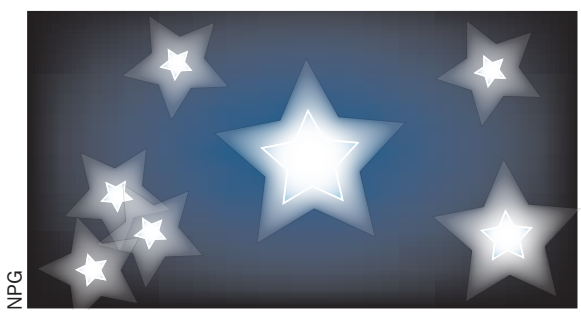

from tumour-resident probiotics, LuGal was converted to luciferin, which was filtered by the kidneys and then excreted in urine. Luciferin could be readily detected in urine using a luciferase assay and, in this new system, the amount of luciferin detected acted as a reporter of burden of liver tumours. Testing as little as $1 \mu \mathrm{l}$ of urine was sufficient to obtain a positive signal, which occurred within $24 \mathrm{~h}$ of probiotic administration. No detrimental health effects were observed in mice $>12$ months after probiotic ingestion.

More work is needed before translation of this new diagnostic approach to the clinic. The researchers are now investigating the trafficking of probiotics from the gut to the liver, as well as exploring other therapeutic and diagnostic probiotic strains.

Katrina Ray

Original article Danino, T. et al. Programmed probiotics fo detection of cancer in urine. Sci. Transl. Med. 7, 289ra84 (2015) 